\title{
Attività di dialisi-vacanze in un centro ospedaliero del sud Italia: Il Centro Dialisi di Maratea
}

\author{
B. Di Iorio, G. Gaudiano, C. Altieri, V. Terracciano \\ Unità Operativa di Nefrologia e Dialisi - Ospedali Unificati del Lagonegrese \\ Lauria $(P z)$
}

\begin{abstract}
Non potrai mai trovare i confini dell'anima neppure se percorrerai tutte le strade che incontri.
\end{abstract}
Eraclito

(da Diogene Laerzio: Vite di filosofi, $X, 7$ )

efficacia delle terapie mediche non può sostenere solo concetti legati alla sopravvivenza, ma deve introdurre esigenze legate alla "qualità di vita" (1-3). Tale termine non è definito da parametri che ne consentano la quantificazione. Ma sicuramente la "qualità di vita" può essere definita in base al Benessere Fisico, al Benessere Emotivo, al Benessere Sociale (4).

Nell'uremico la "quantità di vita" è determinata, sicuramente, anche dalle disposizioni dello Stato che dovrebbe consentire il mantenimento del lavoro o l'erogazione di pensioni di invalidità civile per il dializzato (4), evitare pratiche burocratiche per l'erogazione di farmaci e cure mediche in questi pazienti, accettare che il paziente, anche per scelta emotiva, sia curato da personale di propria fiducia. Accanto a ciò il benessere fisico (percezione soggettiva di essere sano in rapporto all'espletamento delle usuali attività), il benessere emotivo (autostima, grado di contentezza e di piacere di vivere), il benessere sociale (riabilitazione lavorativa, vita sessuale, situazioni coniugali e familiari) trovano una sicura convergenza nella possibilità del paziente uremico di elaborare progetti per il futuro, di predisporre, cioè, il futuro, di programmare, per esempio, periodi di ferie, di vivere, insomma, lontano dalla malattia (almeno nei periodi interdialitici). Questa forte convinzione ci ha "costretti" ad elaborare nel tempo progetti dialisi-vacanze per uremici. Tali progetti (divenuti in seguito veri e propri progettiobiettivi) hanno spinto la USL (oggi ASL) n. 4 del Lagonegrese (Lagonegro$\mathrm{Pz}$ ) a deliberare (n. 386 del 16/4/1993) la creazione di un Centro Dialisi-estiva a Maratea (Pz). Il centro "S. Giuseppe Moscati" degli Ospedali Unificati del Lagonegrese svolge, infatti, dal 19 aprile 1993 attività dialitica routinaria, contumaciale, e attività di dialisi-vacanze.
A quest'ultima attività ha, comunque, concorso negli anni precedenti il Centro Dialisi dell'Ospedale di Lauria (centro principale a cui è aggregato il centro di Maratea) che fin dal 1988 ha ospitato (Tab. I) uremici di altre regioni (e/o nativi della Basilicata residenti in altre regioni) che volessero trascorrrere un periodo di vacanze in Basilicata. È dall'esperienza maturata negli anni precedenti che nasce, poi, il Centro Dialisi-vacanze di Maratea.

Scopo della relazione è la valutazione di 7 anni di attività in questo settore.

TAB. I - ELENCO DELLE REGIONI DI PROVENIENZA DEI PAZIENTI UREMICI IN DIALISI VACANZE

\begin{tabular}{lccccccc}
\hline & 1988 & 1989 & 1990 & $\begin{array}{c}\text { Anni } \\
1991\end{array}$ & 1992 & 1993 & 1994 \\
\hline Campania & 2 & 2 & 7 & 6 & 13 & 16 & 21 \\
Lazio & & 2 & 1 & 2 & 4 & 3 & 4 \\
Puglia & & 1 & 2 & 2 & 2 & 1 & 4 \\
Emilia-Romagna & & 1 & 2 & 1 & 1 & 1 & \\
Lombardia & 1 & 2 & 2 & 5 & 4 & 6 \\
Lucania & 1 & 2 & 3 & 2 & 3 & 4 \\
$\begin{array}{l}\text { Piemonte } \\
\text { Argentina }\end{array}$ & & & 1 & & & & \\
Toscana & & & & 2 & 1 & 3 & 2 \\
Friuli Venezia G. & & & & & & 1 & 1 \\
Umbria & & & & & & & \\
\hline
\end{tabular}




\section{Organizzazione}

Il Centro Dialisi (dotato di 8 posti rene e aumentati a 12 nell'anno successivo) della USL n. 4 del Lagonegrese inizia la sua attività nel 1988 presso l'Ospedale di Lauria. Il personale è attualmente composto da 3 medici, 1 caposala, 13 infermieri professionali, 1 tecnico di emodialisi, 2 ausiliari.

Tale centro (Figg. 1, 2) accanto alla routinaria attività dialitica ha sempre ospitato uremici provenienti da altre regioni per periodi di vacanze. Infatti, nella stessa estate in cui ha iniziato la propria attività, il Centro di Lauria già ospitò 2 uremici in vacanza.

La procedura di accesso al progetto dialisi-vacanze da parte degli ospiti è molto semplice: consiste essenzialmente in una prenotazione (a volte solo telefonica) e all'obbligo della consegna di una impegnativa di richiesta di emodialisi autorizzata dalla USL di appartenenza e dall'esibizione dei markers dell'epatite B e di anti-HCV.

L'attribuzione dei filtri ai pazienti (vedi paragrafo Analisi dei cosi) è stata effettuata tenendo conto di quelli usati dai pazienti nel proprio centro. Ove non fosse disponibile lo stesso filtro se ne è usato uno simile per superficie e identico per tipo di membrana. Inoltre i pazienti ricevevano bicarbonato dialisi o emodiafiltrazione LF o AFB.

A tutti i pazienti veniva effettuata la cinetica dell'urea $(5,6)$ con prelievi di attacco 1 (valutazione generale dei principali parametri bioumorali), stacco (valutazione di BUN, Cr, Na, K, Ca, P, PT) e attacco 2 (stesse valutazione dello stacco). Inoltre in tutti i pazienti veniva riverificata la presenza di HbsAg e antiHCV. Da quel lontano 1988 l'ospitalità di questi pazienti è stata puntualmente favorita negli anni successivi con un incremento di presenze (da varie regioni: Tab. I) e di prestazioni emodialitiche continuo negli anni.

Inoltre (Figg. 1,2) tale attività non ha assolutamente rallentato la routinaria attività dialitica verso i pazienti lucani.

L'incremento delle presenze degli ospiti è avvenuto anche in rapporto alle presenze di pazienti cronici (dal 19\% del 1989 al 40.95\% del 1994); così come le prestazioni emodialitiche erogate (dal $2.51 \%$ del 1989 al 6.08 del 1994).

Questa attività (che comprensibilmente

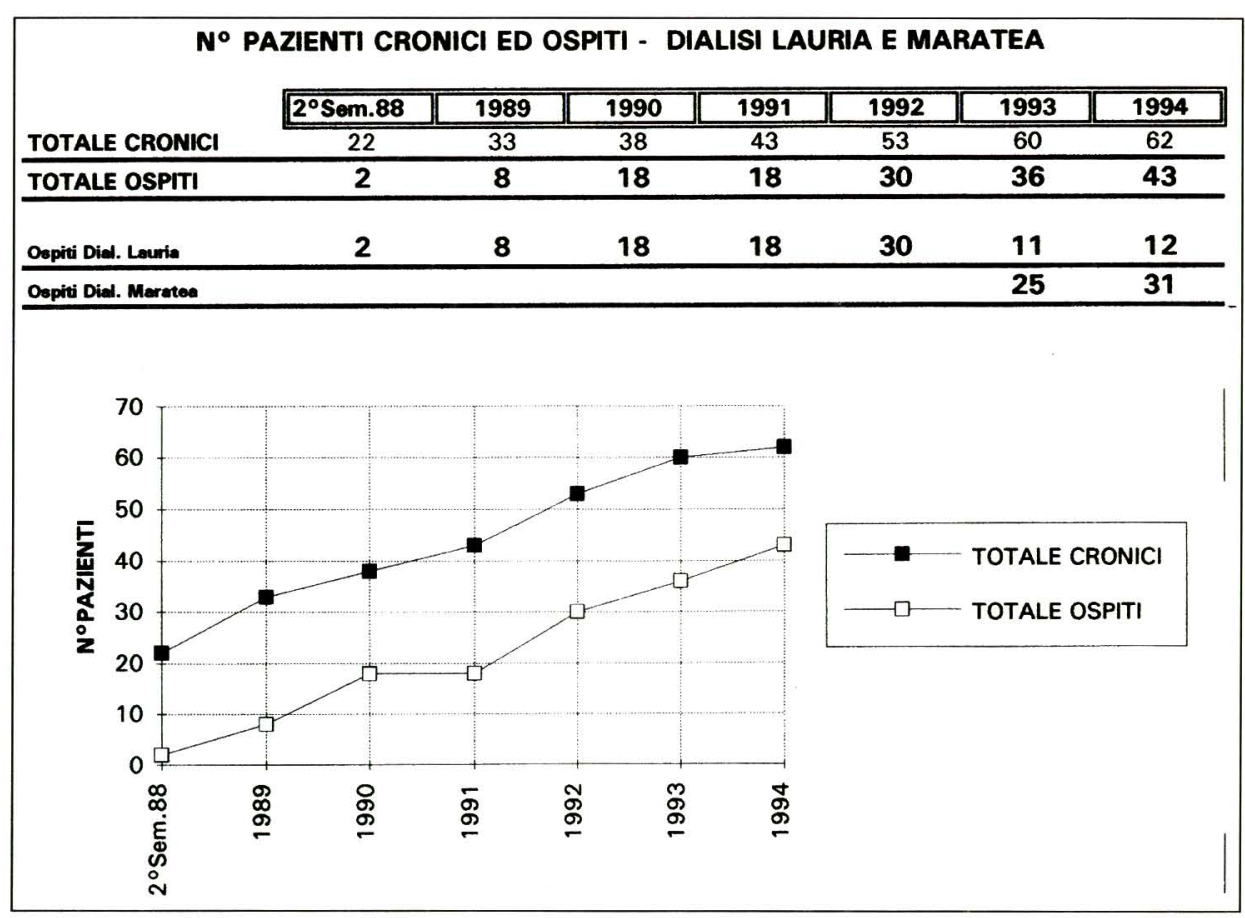

Fig. 1 - Numero di pazienti emodializzati in trattamento continuo presso l'U.O. di Nefrologia e Dialisi degli Ospedali Riuniti del Lagonegrese e numero di pazienti ospitati negli anni di attività del Progetto Dialisi-Vacanze.

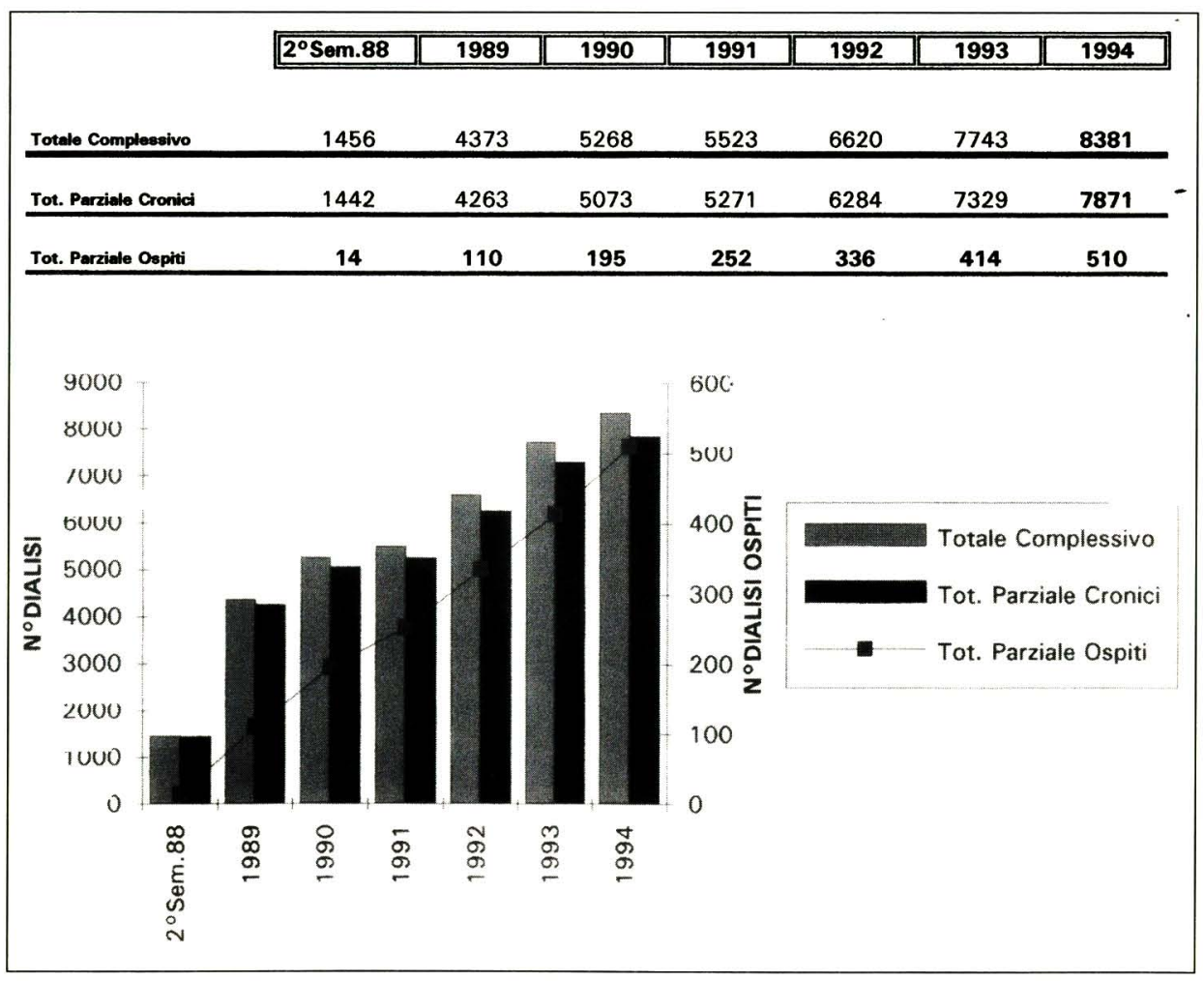

Fig. 2 - Numero di emodialisi effettuate presso l'U.O. di Nefrologia e Dialisi degli Ospedali Riuniti del Lagonegrese ai pazienti locali e a quelli ospitati negli anni di attività del Progetto Dialisi-Vacanze. 
coincide con il periodo di ferie del personale) iniziata spontaneamente ha in seguito necessitato (dato l'alto numero di richieste) di prestazioni in regime di straordinario (1990). In particolare nel 1992 fu necessario istituire un terzo turno serale per gli ospiti.

Il favore con cui l'iniziativa è stata accolta dai pazienti (testimoniata anche, per esempio, dalla presenza ripetuta negli anni successivi degli stessi pazienti anche di regioni lontane) e la propensione culturale di Maratea $(\mathrm{Pz})$ ad iniziative turistiche hanno consentito l'istituzione nel 1993 presso il Plesso Ospedaliero di Maratea degli Ospedali Unificati del Lagonegrese di un Centro Dialisi che svolge attività dialitica routinaria, contumaciale e di dialisi-vacanze.

L'unico fattore che, presumibilmente, ha consentito lo sviluppo di tale attività è l'azione pubblicitaria svolta dai pazienti ospiti presso gli altri uremici del proprio centro dialisi di provenienza.

Il Centro Dialisi di Maratea, risulta dotato di 8 posti rene di cui uno per pazienti HbsAg positivi. Il Centro ha iniziato la sua attività il 19 aprile 1993 con lo stesso personale del Centro Dialisi di Lauria. Ha in dotazione monitor Drake Willock Sistem 1000. Nei mesi di luglio-agosto effettua doppi turni giornalieri, nei restanti mesi ha in gestione 8 pazienti, per cui si effettua un solo turno a giorni alterni.

\section{Analisi dei costi}

Abbiamo effettuato anche un'analisi dei costi relativa alla gestione 1994 (n. pazienti $=43 ;$ n. emodialisi $=510)($ Tab. II).

L'incidenza più elevata in percentuale è data dai costi relativi al personale infermieristico e medico e dai costi dei filtri di dialisi. Le altre voci hanno, poi, incidenze largamente inferiori. Bisogna considerare che il costo del personale è relativo solo alle spese del progetto-obiettivo. Le voci stipendiali, infatti, non sono state considerate in quanto relazionabili alla attività routinaria, che non è certo stata ridotta.

Per quanto riguarda i filtri bisogna precisare che sono stati usati per gli ospiti gli stessi in uso presso il nostro Servizio (senza considerazioni economiche, ma usando gli stessi criteri clinici di attribuzione dei filtri nei nostri pazienti croni-

TAB. II - VALUTAZIONE DEI COSTI TOTALI E PER SINGOLA DIALISI (LIRE) SOSTENUTI NEL 1994 PER IL PROGETTO DIALISI-VACANZE. INCIDENZA PERCENTUALE DI OGNI SINGOLA VOCE

\begin{tabular}{lrrc}
\hline & \multicolumn{1}{c}{ Totale } & Dialisi & Inc \% \\
\hline Medici & 13.160 .000 & 25.804 & 11.57 \\
Infermieri & 37.800 .000 & 74.118 & 33.25 \\
Filtri & 26.170 .500 & 51.135 & 22.94 \\
Soluz. x dialisi & 8.710 .800 & 17.080 & 7.66 \\
Infusioni & 1.213 .800 & 2.380 & 1.07 \\
Linee & 2.535 .000 & 4.970 & 2.23 \\
Aghi fistola & 1.493 .500 & 2.928 & 1.31 \\
Farmaci & 9.282 .000 & 18.200 & 8.16 \\
Presidi & 1.131 .200 & 2.220 & 0.996 \\
Ammort. monitors & 6.800 .000 & 13.300 & 5.96 \\
Acqua & 969.000 & 1.900 & 0.85 \\
Corr. elettrica & 1.020 .000 & 2.000 & 0.897 \\
Esami ematochimici & 352.020 & 6.902 & 0.31 \\
Totale & 113.697 .870 & 222.937 & 100 \\
& & & \\
\hline
\end{tabular}

ci). In particolare l'Hemophan (superfici 1.5 o $1.8 \mathrm{~m}^{2}$ ) nel $24.7 \%$ dei casi; Acetato di Cellulosa $\left(1.4\right.$ o $\left.2.1 \mathrm{~m}^{2}\right)$ nel $53.53 \%$, AN 69 (1.6 m²) nell'1.76\%; Polisulfone L.F. $\left(1.4\right.$ o $\left.1.8 \mathrm{~m}^{2}\right)$ nel $9.21 \%$; PMMA $\left(2.0 \mathrm{~m}^{2}\right)$ nel $10.8 \%$. Tali percentuali sono sovrapponibili a quelle dovute all'uso dei filtri nei pazienti cronici. Inoltre nella valutazione totale dei costi dei filtri è stato tenuto conto anche della sostituzione di n. 8 unità difettose.

Anche nella valutazione dei costi delle linee arteriose e venose e degli aghi-fistola sono state considerate le 15 sostituzioni di linee difettose e le 10 sostituzioni di aghi per punture inefficaci di vasi venosi.

Per quanto riguarda i farmaci il costo totale è riferito all'uso di EPO, carnitina, polivitaminici, eparina, $\mathrm{NaCl}$ iperonico (fiale), plasma-expander. L'EPO da sola incide per il $78.67 \%$ del costo totale di questa voce.

Per i presidi il costo è riferito alle bende elastiche premifistola (che da solo incidono per il 74\%), siringhe, garze, set per infusione, sterilizzanti.

Per le infusioni il costo totale deriva dall'uso di soluzione fisiologica per riempimento delle linee, per la loro eparinizzazione e, durante la dialisi, per il ripristino della volemia del paziente in caso di ipotensione.

Infine la voce ammortamento monitor corrisponde al costo del monitor (consi- derando un' ammortamento in 5 anni, ma valutando solo il bimestre in cui le apparecchiature sono state usate per gli ospiti) e il costo dei pezzi di ricambi usati. È stato valutato anche il costo delle analisi di laboratorio (routinariamente viene effettuata cinetica dell'urea) $(5,6)$. Tale costo appare irrisorio rispetto ai dati di Formica $(7,8)(0.31 \%$ vs 8$)$, ma bisogna considerare che in tali pazienti (gestiti dal nostro Centro per poche settimane) non è stato necessario eseguire esami ormonali e radiografici particolarmente costosi.

Infatti se poi correliamo le altre voci vi è una buona corrispondenza tra le due ricerche; infatti il costo del personale incide per il $44.82 \%$ vs il 41 di Formica, il materiale di dialisi per il $36.19 \%$ vs il 30 , ammortamento monitor per il $6 \%$ vs il 7, i farmaci per 1' $8 \%$ come in Formica $(7,8)$. Il minor costo (circa 222 mila lire vs le 265 di Formica) della dialisi per gli uremici ospiti è probabilmente dovuta alla ridotta incidenza delle spese per il personale che partecipa al progetto-obiettivo (non calcolando l'incidenza stipendiale), al minor costo degli esami ematochimici e all'assenza di costi per esami radiografici.

Nel 1994 un solo paziente è stato ricoverato per 3 giorni per iperkaliemia e durante il ricovero ha effettuato ECG e Rx torace.

Non vi sono state complicanze cliniche 
particolari (pur essendoci tra gli ospiti pazienti a rischio cardiovascolare, pazienti diabetici, pazienti con positività per anti-HCH e 1 paziente con $\mathrm{HbsAg}$ positivo) né dell'accesso vascolare. Nel 1990 vi fu un solo paziente con trombosi acuta della FAV risolta completamente con l'iniezione locale di urokinasi (300 mila unità) e nel 1991 la chiusura di 1 sola FAV con necessità di inserzione di catetere femorale.

\section{Conclusioni}

"La dialisi costituisce il più pesante sacrificio, economico ed organizzativo, che la società sia mai stata chiamata a pagare quale prezzo di una sopravvivenza umana (Regione Piemonte, DPR 23/1980)" (7).

Un così rilevante impegno economico non può esigere soltanto concetti legati alla valutazione dell'efficacia come sopravvivenza, ma deve imporre scelte obbligate che riguardano la "qualità di vita" degli uremici. Tale concetto trova sicuramente una ragione nella correttezza della scelta del tipo di dialisi (e dei materiali usati come valutazione costo/benefici), nella garanzia di un'adeguata e sollecita assistenza medica, nel rispetto dei meccanismi psicologici del paziente, nel sostegno statale per il mantenimento del posto di lavoro o l'erogazione di pensioni di invalidità. Ma "la qualità di vita" è anche impegno attivo dello staff medico per la valorizzazione di atteggiamenti autonomi e alieni da eccessivi sentimenti di passività e rassegnazione da parte dell'uremico.

L'offerta di progetti di dialisi-vacanze, a totale carico del SSN, può rientrare a pieno merito in questo impegno. Nel modello di vita "uomo senza reni" che la dialisi realizza (9), il nefrologo deve ricercare, per quanto possibile, una connessione tra Tecnologia (e la sua razionalità) ed Uremico (con la sua ragione). Un migliore "Umanesimo Scientifico" (9) deve contemplare i termini biologici, i termini economici, i termini clinici (tra i quali non ultima è la qualità di vita del paziente). Tutto quello che può essere fatto (per rendere la vita del paziente in dialisi più vicina possibile ai valori dell'esistenza) dovrebbe essere fatto. Altrimenti i costi economici, anche se contenutissimi, sarebbero inutili, e del tutto effimeri i risultati biologici. Inoltre, soprattutto oggi che orientiamo l'attività medica verso modelli privatistici, non possiamo eludere la nostra attenzione anche da problemi di "marketing" di attività che hanno alla base, prioritariamente, forti motivazioni sociali.

Non trascurabile è, infatti, la valutazione dei costi contenuti di tale attività a fronte del notevole "impatto pubblicitario". La considerazione del notevole richiamo di utenza sanitaria verso la Basilicata, una regione che presenta notevoli flussi di migrazione sanitaria, da altre regioni e con particolare gradimento dell'utenza stessa, non è solo motivo di notevole orgoglio ma indica che la tendenza centrifuga può essere interrotta e invertita.

Infine, alla luce dei DRG, sicuramente il progetto-obiettivo Dialisi-Vacanze è un concetto culturalmente ed economicamente vantaggioso.

\section{Ringraziamenti}

Un particolare ringraziamento va alla caposala (Vigorito I.), agli infermieri professionali (Calderaro R., Cantisani V., Cosentino G., Curzio D., De Lorenzo M.C., Ferrara T., Fittipaldi V., Galardi A., Ielpo E., Perretti E., Romano L., Rossino M., Sassano L.), al tecnico (Limongi $F$.) che hanno reso possibile questi risultati.

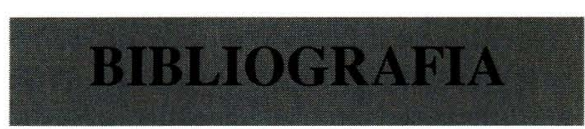

1. Simmons RG, Abress L. Quality-of-life issues for end-stage renal disease patients. Am J Kidney Dis 1990; 3: 201-8.

2. Bremer BA, McCauley CR, Wrona RM, Johnson JP. Quality of life in end-stage renal disease: a reexamination. Am J Kidney Dis 1989; 3: 200-9.

3. Evans RW, Manninen DL, Garrison LP, et al. The quality of life of patients with end-stage renal disease. N Engl J Med 1985; 312: 553-9.

4. Lombardi M, Manfrida GM, Cerrai T, et al. Qualità di vita dell'uremico terminale. Giorn It Nefrol 1994; 11: 227-32.

5. Casino FG, Gaudiano V, Santarsia G, et al. Reconciliation between urea kinetics and direct dialysis quantification in hemodialysis patients. Nephrol Dial Traspl 1991; 10: 801-5.

6. Casino FG. A simple program calculating $\mathrm{Kt} / \mathrm{V}$ and $\mathrm{nPCR}$ without the need for dialyser urea clearance measurement. J Nephrol 1990; 1: 67-8.

7. Formica M, Quarello F, Vanara $\mathrm{F}$, et al. Analisi dei costi della dialisi. Giorn It Nefrol 1991; 8: 167-76.

8. Ragni R, Formica M, Funaro L. Analisi dei costi della dialisi extracorporea. Tecniche Nefrologiche e Dialitiche. Milano: Wichtig ed. 1994; 389-98.

9. Bonomini V. Il pensiero nefrologico nell'evoluzione della terapia renale sostitutiva. Giorn It Nefrol 1993; 10: 331-4. 